\title{
H $\alpha$ variations of the RS CVn type binary ER Vulpeculae
}

\author{
Ö. Çakırlı ${ }^{1}$, C. İbanoğlu ${ }^{1}$, A. Frasca ${ }^{2}$, and S. Catalano ${ }^{2}$ \\ ${ }^{1}$ Ege University Observatory, Bornova, İzmir, Turkey \\ e-mail: ibanoglu@astronomy.sci.ege.edu.tr \\ 2 INAF - Osservatorio Astrofisico di Catania, via S. Sofia 78, 95123 Catania, Italy \\ e-mail: afr@ct.astro.it; scat@ct.astro.it
}

Received 9 September 2002 / Accepted 17 December 2002

\begin{abstract}
We present the results of spectroscopic observations of the active eclipsing binary ER Vul in the 5860-6700 ̊ spectral range carried out at the Catania Astrophysical Observatory. Accurate measurements of radial velocities by using the crosscorrelation technique have been made and a new orbital solution is given. All spectra display chromospheric emission which fills in the $\mathrm{H} \alpha$ absorption lines of both components. The equivalent width $(E W)$ of the $\mathrm{H} \alpha$ emission has been measured by subtraction of a synthetic spectrum built up with spectra of inactive standard stars. The $E W$ of total $\mathrm{H} \alpha$ emission, arisen from both components, shows a phase-dependent variation. It reaches its maximum value just before the primary eclipse. We were able to separate the contributions to the $\mathrm{H} \alpha$ emission from the individual components at phases far from the eclipses. We have found that the secondary, cooler component is the most active and its $\mathrm{H} \alpha$ residual emission shows the same trend as the total $\mathrm{H} \alpha$ emission, along orbital phase. This $\mathrm{H} \alpha$ equivalent width variation can be due to plage-like structures on the chromosphere of secondary star. In addition, a decrease of the $\mathrm{H} \alpha \mathrm{EW}$ around the ingress phase of the primary eclipse is apparent.
\end{abstract}

Key words. stars: activity - binaries: eclipsing - binaries: spectroscopic - stars: individual: ER Vul

\section{Introduction}

ER Vulpeculae (HD 200391, BD $+27^{\circ} 3952$, HIP 103833, $V=$ 7.36, $P=0.698)$ is a double-lined spectroscopic binary (Northcott \& Bakos 1956). Its orbit was first determined by Northcott \& Bakos (1956). Later on the spectroscopic orbit of the ER Vul was re-determined by McLean (1982) and several other radial velocity studies have been performed in recent years (e.g. Hill et al. 1990; Gunn et al. 1996). Northcott \& Bakos (1956) also carried out photometric observations of the system, that allowed them to confirm the eclipses and to present the first preliminary photometric solution. Since then many light curves have been published by Al-Naimiy (1978), Kadouri (1982), Zeilik et al. (1982a,b), Arevalo et al. (1988), Milano et al. (1986), İbanoğlu et al. (1987), Evren (1988), Srivastava et al. (1991), Wunder et al. (1992) and Zeinali et al. (1995). İbanoğlu et al. (1993) found solutions to $B$ and $V$ light curves applying a model that includes proximity effects, wavelike distortions (spots), mutual eclipses and short-term light fluctuations.

Ca II $H$ and $K$ emissions were first detected by Bond (1970). On the basis of the $H$ and $K$ line emission and of the variable light curve, Hall (1976) classified ER Vul as a member of the short-period group of RS CVn systems, with main-sequence

Send offprint requests to: Ö. Çakırlı,

e-mail: cakirli@astronomy.sci.ege.edu.tr components in a detached configuration. However, ER Vul displays a number of interesting peculiarities.

Arevalo et al. (1988) tried to explain the photometric variations and the observed IR and UV excesses as due to a gaseous stream between the two components.

McLean (1982) noticed a variation in the strength of the primary-component's spectral lines which he attributed to circumstellar material around this star with a non-uniform density. Hill et al. (1990) observed small variations (8\%) of the strength of lines of the secondary component between quadratures. They also suggested that, at the epoch of their observations, the secondary component was more active than the primary.

The first study of the $\mathrm{H} \alpha$ line behaviour as a function of orbital phase was made by Barden (1985). He focused on the rotation versus activity relationship among late-type stellar systems. Newmark (1990) presented simultaneous observations in $\mathrm{H} \alpha, \mathrm{H}_{\beta}$, and Ca II (IRT) lines. He found no evidence for extended material around ER Vul in optical spectra as suggested before by Arevalo et al. (1988), while line ratios indicate that chromospheric activity arises primarily in plagelike structures. He also found that the primary component is the more active one. Finally, Lazaro \& Arevalo (1997) carried out low-resolution (2.2 $\AA$ /pixel) spectroscopic observations of ER Vul including $\mathrm{H} \alpha, \mathrm{H}_{\beta}$, and $\mathrm{Ca}$ II (IRT) lines at different orbital phases. In all spectra obtained by them $\mathrm{H} \alpha$ emission from both components appears, after the subtraction of the 
reference spectrum. Equivalent width of the total emission in the $\mathrm{H} \alpha$ line reaches its maximum value during the second photometric maximum $(\phi=0.78)$; however their observations do not fully cover the first maximum. They found clear indication that both components of ER Vul are chromospherically active and no indication, at phases around the two eclipses, of features related to the photometric peculiarities observed by Arevalo et al. (1988). The measured ratio of $\mathrm{H} \alpha$ and $\mathrm{H} \beta$ emission, $E_{\mathrm{H} \alpha} / E_{\mathrm{H} \beta}=1-3$, is indicative of optically thick formation. High resolution spectra $(R=\lambda / \Delta \lambda=52000)$, taken around the primary eclipse, have been presented and analyzed by means of spectral subtraction technique by Gunn \& Doyle (1997). They found excess emission in the cores of $\mathrm{H} \alpha$, Ca II IRT, $\mathrm{Mg}$ I b lines and excess absorption in the helium $\mathrm{D}_{3}$ line. Apart from the detection of a very weak absorption feature $200 \mathrm{~km} \mathrm{~s}^{-1}$ blue-ward of the secondary component, they do not detect spectroscopically the existence of extended regions in this system. They found that chromospheric emission arises in very large plagelike regions. During their observations the secondary component of ER Vul was more active than the primary in $\mathrm{H} \alpha$.

X-ray observations were carried out by Walter \& Bowyer (1981), White et al. (1987) and Kashyap \& Drake (1999). White et al. (1987) found variable X-ray luminosity but no rotational modulation in the X-ray light curve. On the other hand Drake et al. (1986) found that ER Vul was the strongest source among the short-period active binaries in the radio domain. This suggestion has been confirmed by Mutel (1988). The radio light curve obtained by Rucinski (1992) revealed ER Vul as a complex variable.

The two stars which form ER Vul system are approximately twins in mass, radius and luminosity. It is interesting to study this system composed by two solar type stars, which rotate about 40 times faster than the Sun, in order to get a better understanding of magnetic activity phenomena in such extreme cases.

Here we analyze and discuss our spectroscopic observations of ER Vul in a spectral domain including the $\mathrm{H} \alpha$ line and several photospheric lines of interest. The observations and data reduction are described in Sect. 2 . The radial velocity $(R V)$ measurements and the solution of $R V$ curve are presented in Sect. 3. Section 4 is devoted to the analysis of $\mathrm{H} \alpha$ emission.

\section{Observations and data reduction}

The observations have been performed with the echelle spectrograph at $91-\mathrm{cm}$ telescope of the Catania Astrophysical Observatory M. G. Fracastoro station from 10 to 25 August and from 18 to 24 September 2000. The spectra were obtained using the echelle configuration based on a $900-$ lines $/ \mathrm{mm}$ echellette grating as a cross-disperser element and an echelle grating with 79 lines/mm. The spectrograph was fed by the telescope through an optical fiber and was located in a stable position in the room below the dome level. A thinned, back-illuminated CCD with $1024 \times 1024$ pixels (pixel size of $24 \mu \mathrm{m}$ ) has been used during the observing run. We recorded 5 orders in each frame starting from $5860 \AA$ and with a spectral domain of about $850 \AA$. For all the observing runs the width of the slit was set to $550 \mu \mathrm{m}$, projected onto 2.7 pixels of the detector, providing a spectral resolving power $\lambda / \Delta \lambda=14000$.

Typical exposure times for ER Vul spectra were between 1800 and $2400 \mathrm{~s}$. The signal-to-noise ratio $(\mathrm{S} / \mathrm{N})$ attained was about $80-110$ at the continuum near the $\mathrm{H} \alpha$ line, depending on the atmospheric conditions.

In addition to ER Vul, we observed some inactive stars of spectral type similar to that of the components of ER Vul. Among these we have chosen $72 \mathrm{Her}(\mathrm{G} 0 \mathrm{~V})$ to mimic the hotter and cooler components of ER Vul.

Sixteen spectra of ER Vul were collected during the $16 \mathrm{ob}-$ serving nights. The radial-velocity standard star 31 Aql (G7 IV) was nightly observed as well.

The reduction has been performed by using the ECHELLE task of IRAF ${ }^{1}$ (Image Reduction and Analysis Facilities) package following the standard steps:

- background subtraction;

- division by a flat field spectrum given by a halogen lamp;

- wavelength calibration using the emission lines of a thorium-argon lamp;

- normalization to the continuum through a polynominal fit.

The strongest telluric lines are clearly identified in the spectra as instrumental-width features on the continuum and along the rotationally broadened lines of ER Vulpeculae. They were interactively eliminated by a simple linear interpolation while performing the cross-corelation analysis for the radial velocity determinations. We have removed the telluric water vapour lines at the $\mathrm{H} \alpha$ wavelengths using the spectra of Altair (A7 V, $v \sin i=245 \mathrm{~km} \mathrm{~s}^{-1}$ ) acquired during the observing runs. These spectra have been normalized, also inside the very broad $\mathrm{H} \alpha$ profile, to provide valuable templates for the water vapour lines. An interactive procedure, that allows the intensity of the template lines to vary (leaving the line ratios unchanged) until a satisfactory agreement with each observed spectrum is reached, has been applied to correct our ER Vul spectra (see Frasca et al. 2000b).

\section{Radial velocity curves and their solution}

The $R V$ of both components of ER Vul has been the subject of intense studies over many decades by several researchers.

The first radial velocity curves of both components of ER Vul, based on an average of a dozen lines, taken between 1949-1951 at David Dunlap Observatory, were published by Northcott \& Bakos (1956). They found a systemic velocity of $-25.2 \mathrm{~km} \mathrm{~s}^{-1}$ and derived $R V$ curve semi-amplitudes of 138.5 and $149.3 \mathrm{~km} \mathrm{~s}^{-1}$, for the primary and secondary component respectively. The spectroscopic elements obtained by them are given in Table 1 together with the results obtained in subsequent works.

McLean (1982) presented $R V$ observations of ER Vul, based on plates spectra, taken in 1980 at the Haute Provence

\footnotetext{
1 IRAF is distributed by National Optical Astronomy Observatory, which is operated by the Association of University for Research in Astronomy, inc. (AURA) under cooperative agreement with the NSF (National Science Foundation).
} 
Table 1. Comparison of avaliable spectroscopic orbits and new parameters of ER Vul.

\begin{tabular}{lcccc}
\hline \hline Parameter & Northcott \& Bakos & McLean & Hill et al. & Present paper \\
\hline$\gamma\left(\mathrm{km} \mathrm{s}^{-1}\right)$ & $-25.2 \pm 0.8$ & $-23 \pm 3$ & $-24.6 \pm 0.5$ & $-28.3 \pm 3.3$ \\
$K_{\mathrm{p}}\left(\mathrm{km} \mathrm{s}^{-1}\right)$ & $138.5 \pm 1.4$ & $136 \pm 4$ & $139.5 \pm 0.6$ & $139.3 \pm 4.6$ \\
$K_{\mathrm{s}}\left(\mathrm{km} \mathrm{s}^{-1}\right)$ & $149.3 \pm 1.4$ & $139 \pm 2$ & $145.8 \pm 0.5$ & $144.3 \pm 5.2$ \\
$m_{\mathrm{p}} \sin ^{3} i\left(m_{\odot}\right)$ & $0.89 \pm 0.02$ & $0.76 \pm 0.03$ & $0.860 \pm 0.006$ & $0.84 \pm 0.07$ \\
$m_{\mathrm{s}} \sin ^{3} i\left(m_{\odot}\right)$ & $0.83 \pm 0.02$ & $0.75 \pm 0.04$ & $0.823 \pm 0.006$ & $0.81 \pm 0.06$ \\
$a_{\mathrm{p}} \sin i\left(R_{\odot}\right)$ & $1.91 \pm 0.02$ & $1.88 \pm 0.06$ & $1.924 \pm 0.009$ & $1.92 \pm 0.07$ \\
$a_{\mathrm{s}} \sin i\left(R_{\odot}\right)$ & $2.05 \pm 0.02$ & $1.93 \pm 0.04$ & $2.012 \pm 0.007$ & $1.99 \pm 0.08$ \\
$q\left(m_{\mathrm{s}} / m_{\mathrm{p}}\right)$ & $0.93 \pm 0.02$ & $0.98 \pm 0.05$ & $0.957 \pm 0.006$ & $0.96 \pm 0.05$ \\
\hline
\end{tabular}

Observatory and reduced by means of digital techniques. However, the radial velocity curve obtained by McLean was different from those obtained previously by Northcott \& Bakos (1957), and therefore, he found slightly different masses for the component stars.

Further spectroscopic observations based on Reticon detector were made by Hill et al. (1990). They made the observation between 1985 and 1987 at the Dominion Astrophysical Observatory $(D A O)$ with the coudé spectrograph in the spectral domain 4000-4500 $\AA$ and with a spectral purity of about $0.9 \AA$, as they deduced from the FWHM of the emission lines of the comparison lamp. Their orbital parameters are also given in Table 1 . They report the projected rotational velocities of both components, $v \sin i_{\mathrm{H}}=81 \mathrm{~km} \mathrm{~s}^{-1}$ and $v \sin i_{\mathrm{C}}=$ $71 \mathrm{~km} \mathrm{~s}^{-1}$, based on the full width at half maximum of the cross-correlation peaks.

The latest radial velocity curve we found in the literature, was published by Gunn et al. (1996). They observed a group of chromospherically active stars, including ER Vul, in 1994 by using 1.1-meter telescope of Lowell Observatory (Solar Stellar Spectrograph, SSS). The radial velocity data of the two components of ER Vul were determined by the cross-correlation function method.

Our spectra, acquired in two different observing runs, have allowed us to obtain new radial velocities of both components, with a fairly homogeneous coverage of orbital phase. Our $R V$ evaluation has been performed by means of the cross-correlation technique using the IRAF task FXCOR (e.g., Tonry \& Davis 1979; Fitzpatric 1993; Popper \& Jeong 1994). The bright, slow-rotating, radial-velocity standard star $31 \mathrm{Aql}$ (G7IV, $V=5$. 16 ), whose radial velocity is $-100.5 \mathrm{~km} \mathrm{~s}^{-1}$, was used as template for the cross-correlation. We excluded from the analysis the $\mathrm{H} \alpha$ and $\mathrm{Na}_{\mathrm{I}} \mathrm{D}_{2}$ lines which can be contaminated by chromospheric emission and all the spectral ranges heavily affected by telluric absorption lines (e.g. the $\mathrm{O}_{2}$ band 16276-6315). Other less intense and blended telluric lines were interactively taken out by simple linear interpolation, both in ER Vul and 31 Aql spectra.

Radial velocities of the two components, listed in Table 2 together with their standard errors, are weighted averages of the values obtained from the cross-correlation of each order of the target spectra with the corresponding order of the standard spectrum observed in the same night. The usual weight $W_{\mathrm{i}}=\frac{1}{\sigma_{\mathrm{i}}^{2}}$ has been given to each measurement.

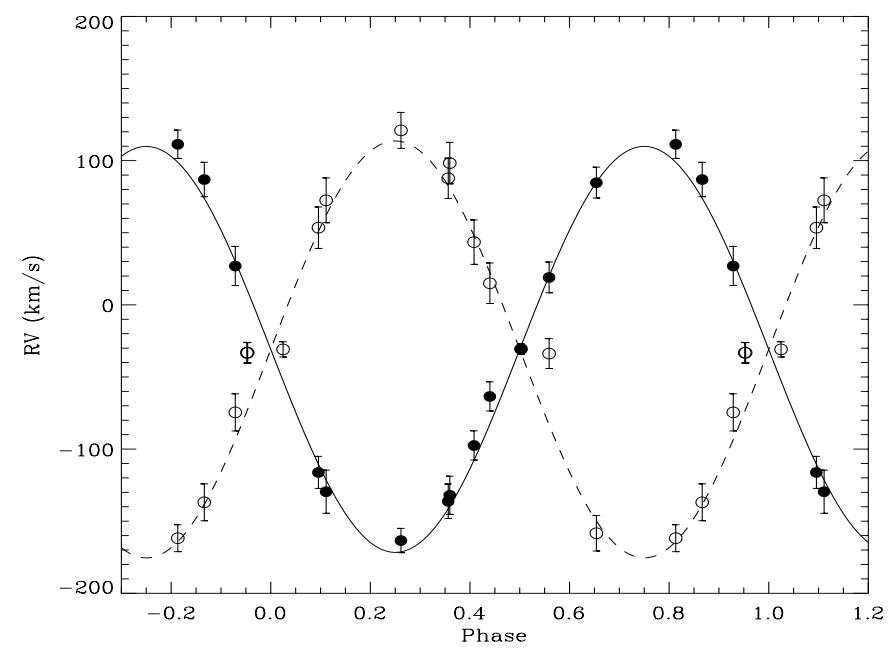

Fig. 1. The $R V \mathrm{~s}$ of the primary and secondary component and their best fits for circular orbit. The phases were computed from the linear ephemeris given in Eq. (1). The empty and filled symbols denote $R V \mathrm{~s}$ of the secondary and primary component, respectively.

The standard errors of the weighted means have been computed on the basis of the errors $\sigma_{\mathrm{i}}$ in the $R V$ values for each order according to the usual formula (see e.g. Topping 1972). The latter $\left(\sigma_{\mathrm{i}}\right)$ are computed by FXCOR according to the fitted peak height and the antisymmetric noise as described by Tonry \& Davis (1979).

The orbital phases have been calculated with the following ephemeris:

$\operatorname{HJD}(\operatorname{MinI})=2449564.31173+0.69809409 \times E$,

where the orbital period is taken from İbanoğlu et al. (1985) and the epoch of primary minimum has been derived from the work of Pribulla et al. (1999). In Fig. 1 the measured radial velocities of both components and their error bars are shown as a function of orbital phase. Dots and circles indicate the velocities of the primary and secondary component, respectively. The solution of $R V$ curves has been determined, assuming circular orbits, by a least-squares fit of all data far from the two eclipses. We have excluded from the fit the points in the phase ranges $0^{\mathrm{p}} .43-0^{\mathrm{p}} .57$ and $0^{\mathrm{p}} .93-1^{\mathrm{p}} .07$. We also remark that our radial velocity curve model does not include non-Keplerian corrections (distortions, Rossiter effect). The sinusoidal $R V$ curves fitted to 
Table 2. Radial velocities of the component stars.

\begin{tabular}{ccrr}
\hline \hline $\begin{array}{c}\text { HJD } \\
2450000+\end{array}$ & Phase & $\begin{array}{c}V_{\mathrm{P}} \\
\left(\mathrm{km} \mathrm{s}^{-1}\right)\end{array}$ & \multicolumn{1}{c}{$\begin{array}{c}V_{\mathrm{S}} \\
\left(\mathrm{km} \mathrm{s}^{-1}\right)\end{array}$} \\
\hline 1767.4569 & 0.450 & $-63.5 \pm 10.1$ & $15.0 \pm 14.1$ \\
1768.4527 & 0.876 & $86.9 \pm 11.9$ & $-136.9 \pm 12.8$ \\
1769.4928 & 0.367 & $-136.2 \pm 12.0$ & $87.8 \pm 14.0$ \\
1771.4159 & 0.121 & $-129.5 \pm 15.0$ & $72.5 \pm 15.5$ \\
1772.4266 & 0.569 & $19.0 \pm 10.7$ & $-33.8 \pm 10.3$ \\
1773.4002 & 0.964 & - & $-33.2 \pm 7.0$ \\
1774.4156 & 0.418 & $-97.5 \pm 10.1$ & $43.5 \pm 15.4$ \\
1776.4759 & 0.962 & - & $-33.2 \pm 7.2$ \\
1775.4932 & 0.370 & $-132.0 \pm 13.2$ & $98.3 \pm 14.4$ \\
1777.4908 & 0.823 & $111.4 \pm 9.8$ & $-161.8 \pm 9.3$ \\
1806.4255 & 0.272 & $-163.4 \pm 8.4$ & $121.0 \pm 12.5$ \\
1807.3977 & 0.664 & $84.8 \pm 10.7$ & $-158.4 \pm 12.3$ \\
1808.4041 & 0.106 & $-116.1 \pm 11.1$ & $53.5 \pm 14.4$ \\
1809.3862 & 0.513 & $-30.6 \pm 3.6$ & - \\
1810.4488 & 0.035 & - & $-30.9 \pm 5.3$ \\
1812.4762 & 0.939 & $27.0 \pm 13.6$ & $-74.5 \pm 12.9$ \\
\hline
\end{tabular}

the data are also displayed in Fig. 1 as solid and dashed lines for the primary and secondary components, respectively.

In Table 1 we compare our orbital parameters with those of Northcott \& Bakos (1956), McLean (1982), and of Hill et al. (1990). Notwitstanding the higher errors, our values of the semi-amplitude of the $R V$ curves are in very good agreement with those derived by Northcott \& Bakos (1956) and by Hill et al. (1990), while the systemic velocity $\gamma$ is slightly lower, but within one sigma.

Since the eclipses are very shallow, the parameters obtained from the light curve analysis have large uncertainties. The inclination of the orbit determined so far is in the $65^{\circ}-72^{\circ}$ range. The solution of the 16 light curves obtained by İbanoğlu et al. (1993) gave a mean value of $66^{\circ} .3 \pm 0.3$. If we use this value for the system inclination, we find for the primary star a mass of $1.09 \pm 0.07 M_{\odot}$, while the mass of the secondary star is $1.06 \pm 0.06 M_{\odot}$. The masses found by McLean are practically equal to one solar mass and result to be systematically smaller than all the other determinations that are, instead, in fairly good agreement between themselves. We found an orbital separation of $4.27 R_{\odot}$. Combining this value with the fractional radii of the components found by İbanoğlu et al. (1993), we derived the radii of both components as $\simeq 1.2 R_{\odot}$. The absolute parameters of the components are presented in Table 3 . The results indicate that both components of ER Vul have nearly equal masses and radii.

\section{Analysis of the $\mathrm{H} \alpha$ line}

Since we cannot directly image the stellar surface, with its photospheric and chromospheric inhomogeneities, we must rely on indirect methods and theoretical models to extract the needed information from light-curves and/or spectral-line fluxes.

The $\mathrm{H} \alpha$ line is an important indicator of chromospheric activity. Only the very active binaries show $\mathrm{H} \alpha$ emission always above the continuum (e.g. II Peg, V711 Tau, UX Ari, XX Tri, AR Psc); in less active stars only a filled-in absorption line is observed. For some objects the $\mathrm{H} \alpha$ line goes from filled-in
Table 3. The average physical parameters and orbital elements for ER Vul, adopted from İbanoğlu et al. (1993) and the present $R V$ solution.

\begin{tabular}{ccc}
\hline \hline Parameter & Hot & Cool \\
\hline$m / m_{\odot}$ & $1.09 \pm 0.07$ & $1.06 \pm 0.06$ \\
$R / R_{\odot}$ & $1.16 \pm 0.06$ & $1.18 \pm 0.14$ \\
$L / L_{\odot}$ & $1.56 \pm 0.15$ & $1.52 \pm 0.37$ \\
$M_{\text {bol }}$ & 4.28 & 4.31 \\
$T(\mathrm{~K})$ & 6000 & $5883 \pm 52$ \\
\hline$i$ & $66.32 \pm 0.35$ \\
$a / R_{\odot}$ & $4.27 \pm 0.10$ & \\
$P$ (days) & 0.69809409 & \\
\hline
\end{tabular}

absorption to emission during flare events (Catalano \& Frasca 1994) or during activity variations over longer time scales, like those induced by rotational modulation or cycles.

The $\mathrm{H} \alpha$-line analysis is more difficult in a double-lined system in which both spectra are simultaneously seen and shifted at different wavelengths, according to the orbital phase. Therefore, in order to extract a valid information about the chromospheric contribution, a comparison with a synthetic spectrum constructed with two stellar spectra that mimic the two components of the system in absence of activity is needed.

As shown by several investigators (e.g. Barden 1984; Montes et al. 1994; Frasca \& Catalano 1994; Frasca et al. 2000a), spectral subtraction is a suitable technique for the study of stellar activity because it allows to isolate the chromospheric signature in a spectral line, to identify the star which is responsible for the chromospheric emission in double systems and to obtain a more secure integration of the emission contribution in the residual spectra.

In addition to the sensitivity to chromospheric layers, $\mathrm{H} \alpha$ emission is also a good diagnostic of inter-system matter in the form of gas streams, transient or classical accretion disks and rings in mass-transferring binaries like those of the Algol type, in which the cooler star fills-in its Roche lobe and transfers mass to the hot companion (see e.g. Richards \& Albright 1999). Evidence for inter-system matter, emitting and absorbing at $\mathrm{H} \alpha$ wavelengths, has been also recently reported by Marino et al. (2001) for HR 7428, a long-period, detached atypical RS CVn binary composed of a bright K-type giant and an A-type star. Furthermore, extended, prominence-like structures producing extra-absorption at $\mathrm{H} \alpha$ wavelength have been detected in some eclipsing RS CVn systems by Hall \& Ramsey (1992) and by Frasca et al. (2000a).

The two components of ER Vul, even if classified as G0V and G5V (Northcott \& Bakos 1956), should have effective temperatures much closer to each other, as can be inferred from a recent light curve solution (İbanoğlu et al. 1993) which gives values of $T_{\text {eff }}=6000,5883 \mathrm{~K}$ for the primary and secondary component, respectively. For main-sequence stars these temperatures correspond to F9V and G0V spectral types, respectively, according to the calibration reported by Gray (1992).

For this reason we have chosen 72 Her as a reference inactive star to simulate both components of the system. 72 Her is a G0V star with a very low activity degree, as can be deduced from the low value of the Ca II $S$ index of the Mt. Wilson 
spectrophotometer (Duncan et al. 1991) and from the low $\mathrm{H} \alpha$ residual flux, in comparison with other single $\mathrm{G}$ stars (Herbig 1984).

We have taken many exposures of $72 \mathrm{Her}$, and we have chosen, for each observing run, the best spectrum as template. In order to reproduce the shape of the $\mathrm{H} \alpha$ line, the template spectrum must be broadened to simulate the rotation of the ER Vul components. Hill et al. (1990) have measured $v \sin i$ 's of both component of ER Vul from the FWHM of the peaks that they produce in the cross-correlation function, and found average values of $81 \mathrm{~km} \mathrm{~s}^{-1}$ for the primary component and $71 \mathrm{~km} \mathrm{~s}^{-1}$ for the secondary one. We have checked the consistency of these measurements with the values of the projected rotational velocity that can be deduced from the photometric and orbital solution in the hypothesis that both star are synchronously rotating. From the fractional radii and system inclination given by İbanoğlu et al. (1993) and from our own $R V$ solution we find $v \sin i=77 \mathrm{~km} \mathrm{~s}^{-1}$ for the primary component and $v \sin i=78 \mathrm{~km} \mathrm{~s}^{-1}$ for the secondary one. These values, that agree fairly well with those measured by Hill et al. (1990), have been used by us to generate the synthetic spectrum.

The relative contribution of each component to the observed spectrum has been evaluated through the radii and effective temperatures listed in Table 3. We have also taken into account the variation of the relative contributions during the eclipses and the distortion produced by the eclipses on the line profiles of the eclipsed star following the same procedure used by Frasca et al. (2000a) for the analysis of AR Lac spectra. The spectra of the primary and secondary star in the synthetic spectrum have been wavelength shifted according to our $R V$ solution presented in Sect. 3. To define the net $\mathrm{H} \alpha$ emission of the two components we have subtracted to each spectrum of ER Vul the synthetic one at the corresponding phase. In the residual spectra, obtained by the subtraction, the absorption lines cancel out and the excess emission of the two components in the $\mathrm{H} \alpha$ core appears as a double peak well above the noise (see Fig. 2).

The net equivalent width of the "total" $\mathrm{H} \alpha$ emission $\left(E W_{\mathrm{TOT}}\right)$ has been evaluated on the difference spectra integrating along the entire residual emission profile. This cumulative integration has the drawback to merge the emission contribution of both stars, but it can be done at any phase, even when the two residual $\mathrm{H} \alpha$ lines are too much blended to be separated. The errors on the measured $E W_{\text {TOT }}$ values were estimated as the product of the inverse of $\mathrm{S} / \mathrm{N}$ ratio, evaluated in two windows on the right- and left-hand side of the $\mathrm{H} \alpha$ line, and the width of the integration range. $E W_{\mathrm{TOT}}$ values, together with their corresponding heliocentric JD, phase, and spectral weight of the secondary component $\left(W_{\mathrm{C}}\right)$, are listed in Table 4 .

These $E W_{\text {TOT }}$ values are relative to the local continuum that is a mixture of the primary and secondary star spectra whose weights vary during the eclipses, affecting the measured equivalent width. However, a smooth variation of $E W_{\text {TOT }}$, with a maximum just before phase $0^{\mathrm{p}} .0$ can be easily seen in Fig. $3 \mathrm{c}$.

In addition, a slight decrease of $E W_{\mathrm{TOT}}$ around the ingress of the primary eclipse can be noticed. Three spectra at about the same phase $\left(\phi \simeq 0^{\mathrm{p}} .95\right)$ obtained in different nights lead to $E W_{\text {TOT }}$ values in good agreement among themselves, and
Table 4. $\mathrm{H} \alpha$ equivalent widths measurements of ER Vul obtained in 2000.

\begin{tabular}{ccccccc}
\hline \hline $\begin{array}{c}\text { HJD } \\
2451000+\end{array}$ & Phase & $W_{\mathrm{C}}$ & $\begin{array}{c}E W_{\text {TОт }} \\
(\AA)\end{array}$ & $\begin{array}{c}\text { Error } \\
(\AA)\end{array}$ & $\begin{array}{c}E W_{\mathrm{P}} \\
(\AA)\end{array}$ & $\begin{array}{c}E W_{\mathrm{S}} \\
(\AA)\end{array}$ \\
\hline 767.45686 & 0.450 & 0.476 & 0.316 & 0.060 & - & - \\
768.45267 & 0.876 & 0.490 & 0.648 & 0.086 & 0.551 & 0.810 \\
769.49284 & 0.367 & 0.490 & 0.409 & 0.083 & 0.358 & 0.500 \\
771.41592 & 0.121 & 0.490 & 0.558 & 0.078 & 0.490 & 0.723 \\
772.42660 & 0.569 & 0.490 & 0.189 & 0.100 & - & - \\
773.40020 & 0.964 & 0.526 & 0.399 & 0.070 & - & - \\
774.41560 & 0.418 & 0.490 & 0.424 & 0.074 & 0.551 & 0.342 \\
775.49315 & 0.962 & 0.523 & 0.330 & 0.059 & - & - \\
776.47593 & 0.370 & 0.490 & 0.537 & 0.088 & 0.487 & 0.586 \\
777.49082 & 0.823 & 0.490 & 0.549 & 0.125 & 0.414 & 0.772 \\
806.42552 & 0.272 & 0.490 & 0.514 & 0.102 & 0.526 & 0.620 \\
807.39765 & 0.664 & 0.490 & 0.554 & 0.083 & 0.354 & 0.837 \\
808.40410 & 0.106 & 0.490 & 0.547 & 0.077 & 0.520 & 0.518 \\
809.38623 & 0.513 & 0.426 & 0.387 & 0.043 & - & - \\
810.44884 & 0.035 & 0.529 & 0.633 & 0.084 & - & - \\
812.47615 & 0.939 & 0.492 & 0.365 & 0.050 & - & - \\
\hline
\end{tabular}

about $0.3 \AA$ lower than the other data at near phases. It is interesting to notice that the $E W_{\text {TOT }}$ value at phase $0^{\text {p }} .035$, near the eclipse egress, is not affected by this decrease. A possible explanation for this behaviour could be that the extended atmosphere of the more active, secondary star is seen projected against the primary star disk at these phase producing an extra absorption in the $\mathrm{H} \alpha$ line. A similar effect was observed in AR Lac (Frasca et al. 2000a) and in some other RS CVn eclipsing systems (Hall \& Ramsey 1992). Another possible scenario is the presence of one large active region on the pole of the primary component that is almost fully occulted during the primary eclipse. Such a high-latitude structure would not produce any rotational modulation of $\mathrm{H} \alpha$ emission of the primary component, in agreement with our observations (see next sentences). In addition, the value of $E W_{\text {TOT }}$ at these phases is nearly equal to that of the secondary star only, taking into account the light ratio, thus indicating that the emission from the primary star is either fully absorbed or completely eclipsed.

The range of our $E W_{\text {TOT }}$ variation is between 0.19 and $0.65 \AA$. Lázaro \& Arévalo (1997) observed a range of variation of total $\mathrm{H} \alpha E W$ from $0.43 \AA$ (near the secondary eclipse) to $0.89 \AA$ (around phase 0 p.75). Notwithstanding the incomplete phase coverage of their observations, the shape of their $\mathrm{H} \alpha E W$ curve, with a maximum around the second quadrature, is a bit different from our own. A common feature to both data sets is the decrease of $\mathrm{H} \alpha$ emission during the primary eclipse. Furthermore, they observed a steady decrease of $\mathrm{H} \alpha$ emission from $\phi=0$ p.9 to $\phi=1^{\mathrm{p}} .0$. The average value of the $\mathrm{H} \alpha$ excess $E W$ at phase $\sim 0.0$ reported by Gunn \& Doyle (1997), $0.596 \AA$, is comparable with the value observed by us around the primary eclipse, with the exception of our data at phase $\simeq 0$ p.96 for which we found $E W_{\text {TOT }} \sim 0.35 \AA$. No variation of the residual $\mathrm{H} \alpha E W$ from $\phi=0^{\mathrm{p}} .94$ to $\phi=1^{\mathrm{p}} .05$ is visible in their observations.

Unfortunately, we have no photometric light curve obtained simultaneously. Therefore we can not compare the behaviour of the $\mathrm{H} \alpha$ emission with that displayed by the photometry. 

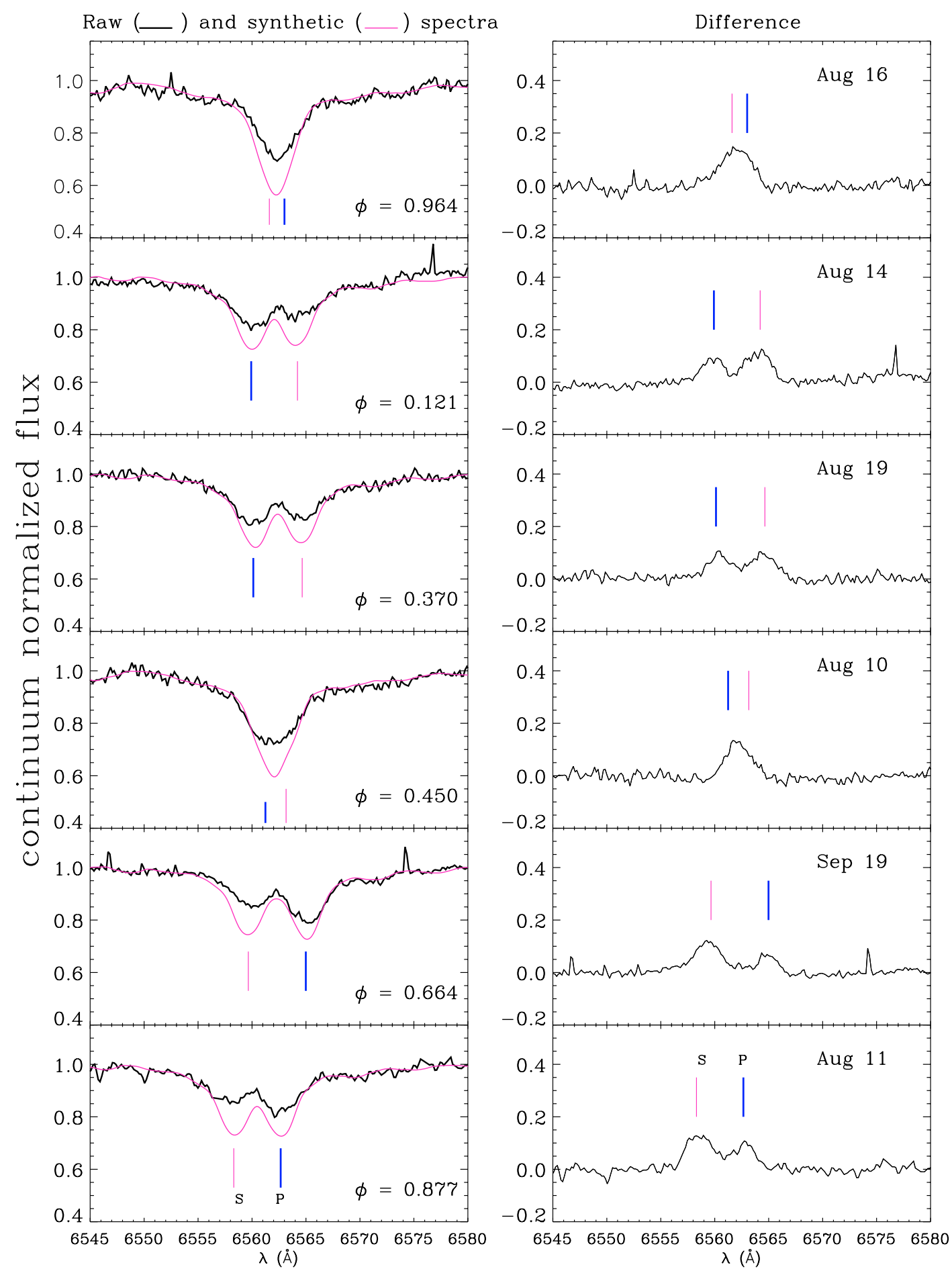

Fig. 2. Sample of $\mathrm{H} \alpha$ spectra of ER Vul. In the left panels the observed spectra are displayed by a thick line, while the synthetic ones are reproduced by thin lines. In the right-side panels the differences are shown. The phases of observations and the wavelength of the H $\alpha$ centers of the more massive (P) and less massive (S) component are also indicated by thick and thin marks, respectively.

Since $\mathrm{H} \alpha$ emission from both components has been seen in all spectra in which they are well resolved, we have extracted the emission $E W$ of each component by a fit of two Gaussians to the residual $\mathrm{H} \alpha$ profile.

These $E W \mathrm{~s}$ are measured with respect to the "total" continuum that contains the contribution of both stars. In order to obtain the true equivalent width of each component we have divided these $E W$ values by the contribution to the continuum of the corresponding star. These corrected equivalent widths, $E W_{\mathrm{P}}$ and $E W_{\mathrm{S}}$, are listed in Table 4 and have been plotted in Figs. 3d, 3e. Notwithstanding the scatter of data, the secondary component appears to be the more active and the more variable one, with a maximum emission between phase $0^{\mathrm{p}} .7$ and 0 .9. The behaviour of $E W_{\mathrm{S}}$ as a function of phase, displayed in Fig. 3e, follows that of $E W_{\text {TOT }}$, while the primary star shows a nearly constant trend. 

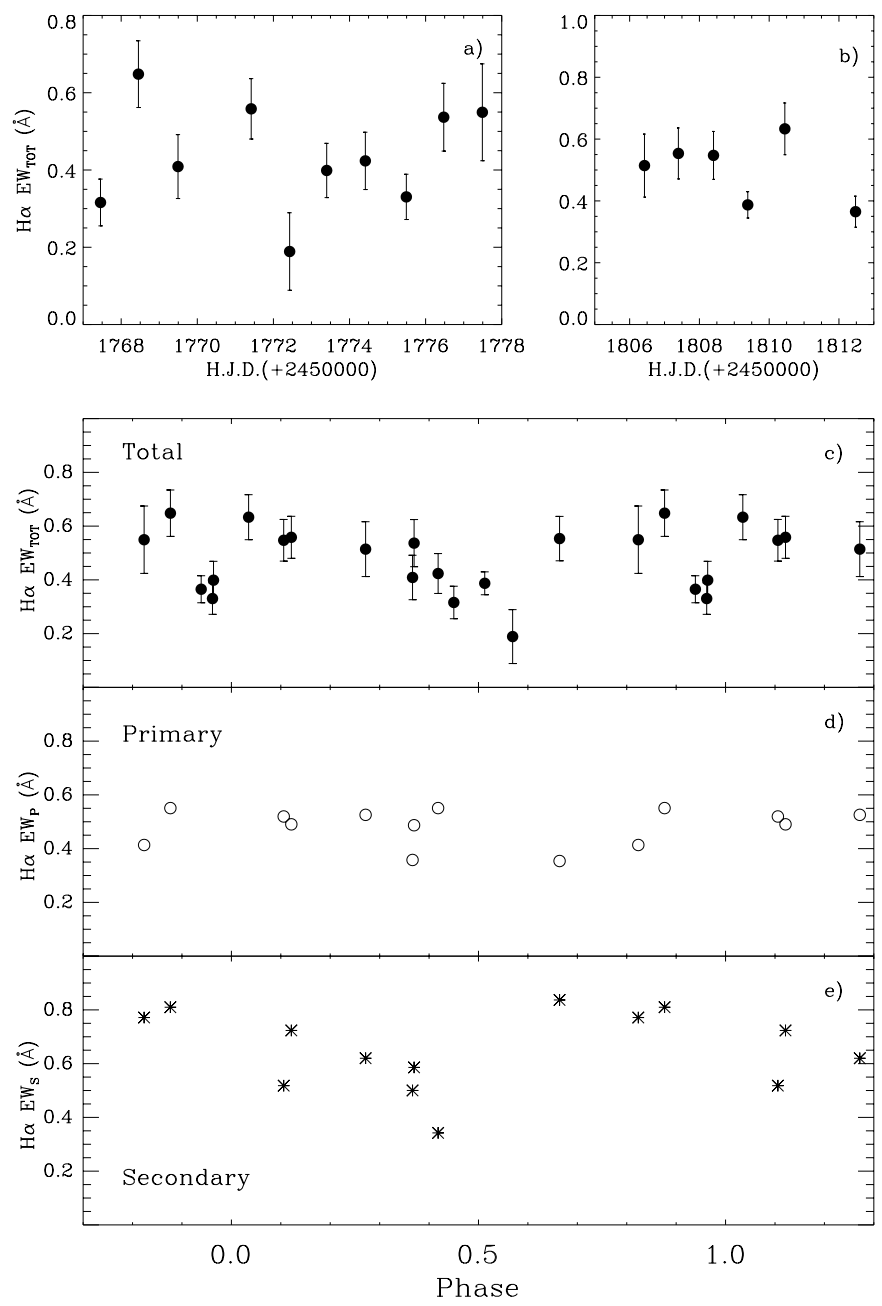

Fig. 3. Equivalent width of $\mathrm{H} \alpha$ total emission of ER Vul versus time (a) and b)) and phase (c)). The equivalent width of the primary and secondary component, $E W_{\mathrm{P}}$ and $E W_{\mathrm{S}}$, are shown as a function of the orbital phase in $\mathbf{d}$ ) and $\mathbf{e}$ ), respectively.

The average intensity of the $\mathrm{H} \alpha$ emission of the secondary star is generally greater than the primary one, by about a factor of 1.5, also taking into account the relative contributions. The same behaviour was observed by Hill et al. (1990) and by Gunn \& Doyle (1997), but the opposite has been reported by Barden (1985), who found instead $E W_{\mathrm{P}}=0.466 \AA$ and $E W_{\mathrm{S}}=0.204 \AA$, and by Newmark (1990). Hence the activity levels of the components of ER Vul appear to be variable in time.

\section{Conclusions}

In this paper we have presented a spectroscopic study of the short-period RS CVn type eclipsing binary ER Vul. We have obtained the orbital elements of the system by analysing the radial velocity curve. The results are in agreement with those obtained by Northcott \& Bakos (1957) and by Hill et al. (1990). However, the masses of the components derived by us are larger than those obtained by McLean (1982), but are well consistent with those derived by Northcott \& Bakos (1956) and by Hill et al. (1990).
A set of spectra with moderate spectral resolution obtained with a good phase coverage has allowed us to study the behaviour of the the $\mathrm{H} \alpha$ line of the components of ER Vul as a function of orbital phase. We concluded that both components of ER Vul are chromospherically active stars. However, during our observing run the secondary component appears more active than the primary. The same was observed by Hill et al. (1990) and by Gunn \& Doyle (1997), but the opposite behaviour has been reported by Barden (1985) and by Newmark (1990). Hence the activity levels of the components of ER Vul appear to be variable in time.

A significant result of this work is the detection of variability of the $\mathrm{H} \alpha$ emission $E W$ with the orbital phase. The total $\mathrm{H} \alpha$ emission excess, $E W_{\text {TOT }}$, shows a maximum around phase $0^{\mathrm{p}} .9$ and a minimum around phase $0^{\mathrm{p}} \cdot 6$. A very similar trend is displayed by the $\mathrm{H} \alpha$ emission of the secondary component, $E W_{\mathrm{S}}$, while a rather flat behaviour is shown by the $\mathrm{H} \alpha$ equivalent width of the primary star, $E W_{\mathrm{P}}$, as a function of the orbital phase. These variations could be indicative of the presence of chromospheric active regions on the secondary component, in addition to an average basic emission.

A slight decrease of $E W_{\text {TOT }}$ has been also observed at phases near to the first contact of the primary eclipse $(\phi=0$ p.95). An explanation of this effect in terms of extraabsorption produced by a prominence-like structure connected to the cool secondary component, similar to that found in other RS CVn systems (Hall \& Ramsey 1992; Frasca et al. 2000a), is proposed.

We have found that the activity levels of the components of ER Vul are variable also on long time scales so that at different epochs the average activity level of the system appears significantly different. We have discussed here the $\mathrm{H} \alpha$ emission behaviour of ER Vul on a short time-scale (as the two observing runs in 2000). Simultaneous photometric and spectroscopic observations are desirable for clarifying the activity levels of the components and also for better understanding short-time variations of light curve and spectral lines. Those observations would also allow a more precise determination of the location of the active regions in each star.

Acknowledgements. We gratefully aknowledge the referee, Dr. Ribas, for his very useful suggestions. Ö. Çakırlı thanks Prof. M. Rodonò for his hospitality and allowance of telescope time for the observations. He is grateful to Turkish Scientific and Research Council of Turkey and for their financial support. He is also grateful to Koç VAKFI for his educational-background supporting. We are also grateful to Daniela Recupero for the careful reding of the manuscript. This work has been supported by the Italian Ministero dell'Istruzione, Università e Ricerca (MIUR) and by the Regione Sicilia which are gratefully acknowledged. This work was partly-supported by Ege University Science Fund under the project number 2000/FEN/013. This research has made use of the ADS-CDS databases, operated at the CDS, Strasbourg, France.

\section{References}

Al-Naimiy, H. M. 1978, IBVS, 1418

Arevalo, M. J., Lazaro, C., \& Fuensalida, J. D. 1988, AJ, 96, 1061

Barden, S. 1985, ApJ, 295, 162 
Bond, H. E. 1970, PASP, 82, 321

Catalano, S., \& Frasca, A. 1994, A\&A, 287, 575

Evren, S. 1988, Ap\&SS, 140, 125

Fitzpatrick, M. J. 1993, in Astronomical Data Analysis Software and System II, ed. R. J. Hanish, R. V. J. Brissenden, \& J. Barnes, PASPC, 52, 472

Fraquelli, D. A. 1984, ApJ, 276, 243

Frasca, A., \& Catalano, S. 1994, A\&A, 284, 883

Frasca, A., Marino, G., Catalano, S., \& Marilli, E. 2000a, A\&A, 358, 1007

Frasca, A., Freire Ferrero, R., Marilli, E., \& Catalano, S. 2000b, A\&A, 364, 179

Gunn, A. G., Hall, J. C., Lockwood, G. W., \& Doyle, J. G. 1996, A\&A, 305,146

Gunn, A. G., \& Doyle, J. G. 1997, A\&A, 318, 60

Hall, D. S. 1976, Proc. IAU Coll. No. 29, Budapest, Part 1, 287

Hall, J. C., \& Ramsey, L. W. 1992, AJ, 104, 1942

Hill, G., Fisher, W. A., \& Holmgren, D. 1990, A\&A, 238, 145

İbanoğlu, C., Akan, M. C., Evren, S., \& Tunca, Z. 1985, IBVS, 2782

İbanoğlu, C., Evren, S., \& Tunca, Z. 1987, Ap\&SS, 136, 225

İbanoğlu, C., Evren, S., Akan, M. C., Tunca, Z., \& Keskin, V. 1993 , A\&A, 269, 310

Kadouri, T. H. 1982, IBVS, 2057

Kashyap, V., \& Drake, J. J. 1999, ApJ, 524, 988

Lazaro, C., \& Arevalo, M. S. 1997, AJ, 113, 2283

Marino, G., Catalano, S., Frasca, A., \& Marilli, E. 2001, A\&A, 375, 100
McLean, B. J. 1982, MNRAS, 201, 421

Milano, L., Mancuso, S., Vittone, E., \& D’Orsi, A. 1986, Ap\&SS, 124, 83

Mutel, R. L. 1988, IAUS, 129, 73

Newmark, K. J. S. 1990, Ph.D. Thesis, Penn. State University

Nortchott, R. J., \& Bakos, G. A. 1956, AJ, 61, 188

Nortchott, R. J., \& Bakos, G. A. 1957, AJ, 72, 89

Popper, D. M., \& Jeong, Y. C. 1994, PASP, 106, 184

Pribulla, T., Chochol, D., \& Parimucha, S. 1999, IBVS, 4751

Richards, M. T., \& Albright, G. E. 1999, ApJS, 123, 537

Rucinski, S. M. 1992, PASP, 104, 1177

Shengbang, Q., Qingyao, L., \& Yulan, Y. 1997, Ap\&SS, 257, 1

Simkin, S. M. 1974, A\&A, 31, 129

Srivastava, R. K., Padalia, T. D., \& Srivastava, J. B. 1991, Ap\&SS, 182,281

Topping, J. 1972, Errors of Observation and Their Treatment (Chapman and Hall Ltd.), 89

Tonry, J., \& Davis, M. 1979, AJ, 84, 1511

White, N. E., Culhane, J. L., Parmar, A. N., \& Sweeney, M. A. 1987, MNRAS, 227, 545

Walter, F. M., \& Bowyer, S. 1981, ApJ, 245, 671

Wunder, E. 1992, IBVS, 3760

Zeilik, M., Elston, R., Henson, G., Schmolke, P., \& Smith, P. 1982a, IBVS, 2107

Zeilik, M., Baca, B., Batuski, B., et al. 1982b, IBVS, 2221

Zeinali, F., Edalati, M. T., \& Mirtorahi, M. T. 1995, IBVS, 4190 